\title{
HARDENING OF FCC CRYSTALS UNDERGOING MULTIPLE SLIP
}

\section{JOHN L. BASSANI}

\section{Department of Mechanical Engineering and Applied Mechanics} University of Pennsylvania, Philadelphia, PA 19104, USA

\section{INTRODUCTION}

Based on the recent experiments of Wu, Bassani and Laird (1989) and other observations dating back 35 or more years, a new analytical characterization for the evolution of the instantaneous hardening moduli $\left\{h_{\alpha \beta}\right\}$ for a single crystal undergoing multiple slip plastic deformation has been developed by Bassani and Wu (1989) (see also Bassani, 1990). These moduli relate the rate of hardening of the critical shear stress (or yield stress) on each slip system to the rates of slip (shear) on all $\mathbf{N}$ systems as

$$
\dot{\tau}_{\mathrm{cr}}^{(\alpha)}=\sum_{\beta=1}^{\mathrm{N}} \mathrm{h}_{\alpha \beta} \dot{\gamma}^{(\beta)}
$$

The new description of the hardening moduli incorporates the effects of slips on all systems on the active hardening of each system. The notion of truly latent hardening is reinterpreted within the framework of incremental plasticity. It is found that an accurate characterization of hardening is obtained even when the off-diagonal components of $h_{\alpha \beta}$ vanish, which assures positive definiteness of $\left\{\mathrm{h}_{\alpha \beta}\right\}$ so long as no slip system softens. In this limit, a previously latent system only hardens once it is activated. Predictions of Bassani and $\mathrm{Wu}(1989)$ based on this purely active-hardening law are in excellent agreement with observations for FCC crystals under uniaxial stress, including the dependence of hardening on tensile axis orientation and on strain, lattice rotations and tensile overshoot, and latent hardening.

Simple forms for $h_{\alpha \beta}$ that have been widely adopted typically take active hardening on any slip system (diagonal components $h_{\alpha \alpha}$ ) to be independent of the slips on other systems and, thereby, incorporate only a limited coupling between slip systems through off-diagonal components that scale with diagonal components. As a consequence, these simple descriptions cannot account for stage I easy glide followed by stage II rapid hardening associated with secondary slips and the significant differences in overall hardening observed for different orientations of the tensile axis (see, e.g., Diehl, 1956 and Honeycombe, 1984, Chap. 4). These behaviors, as typically observed, are predicted in Fig. 1 for copper based on the new hardening law described below. Other behaviors that are not captured by the simple hardening descriptions include the significant amount of secondary slips before overshoot (Basinski and Basinski, 1970; Joshi and Green, 1980), many of which are on the order of one-tenth of the primary slip, and the high "latent hardening" at the onset of secondary deformation observed by Franciosi, et al. (1980).

The orientations numbered 2,3 and 4 in Fig. 1 are so-called "single slip" orientations in the sense that one slip system is stressed significantly higher 
than all others while orientations 1 and 5 lead to equal slip on several systems. In the "single slip" orientations the initial hardening is low (stage I easy glide) so long as only one system is active and then it dramatically increases as other systems become active (stage II), while the extent of easy glide depends on orientation. Furthermore, even though eight systems slip equally for the [100] orientation 5 the hardening is lower than that for the [111] orientation 1 that involves equal slip on six systems. These behaviors point to the fact that different pairs of systems interact differently and, therefore, cannot be captured by the simple hardening rules.

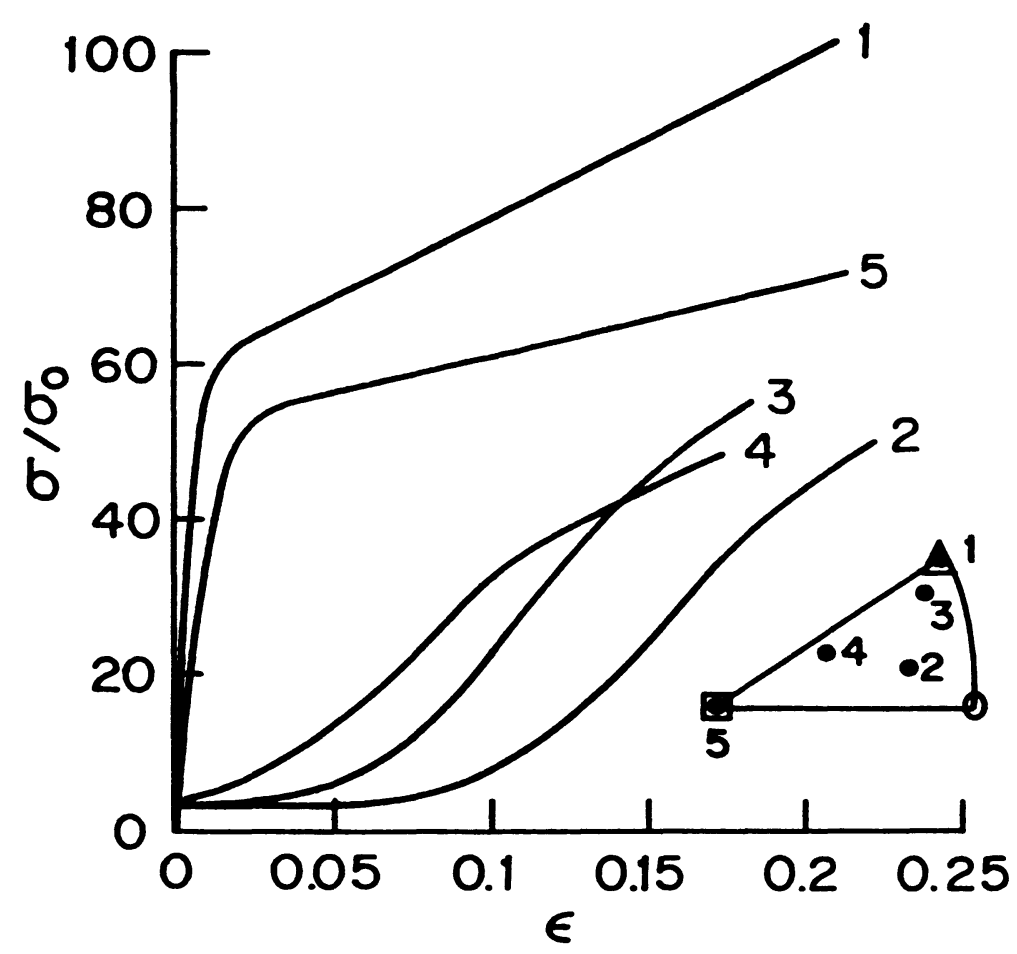

FIG. 1. Typical uniaxial stress-strain curves for various orientations of the tensile axis in FCC crystals. Note the significant variations in hardening. From the numerical simulations based on the new hardening law.

Wu, Bassani and Laird (1989) have noted that many of the observed phenomena caused by slip interactions often have been misinterpreted as intrinsic characteristics of single-slip. One prominent example is that the notion of a stronger (truly) latent-hardening is actually a rapid active-hardening caused by slip interactions when a secondary system becomes highly stressed. This high hardening rate associated with slip interactions must be accounted for. The importance of slip interactions is also revealed by other observations, for example, stage II rapid hardening is always accompanied by secondary slip(s) and as the initial orientation of the tensile axis approaches the [100]-[111] symmetry boundary the hardening rate increases in a way consistent with the multi-slip condition.

Based on these observations, inequality restrictions on the instantaneous hardening moduli $h_{\alpha \beta}$ have been derived by $\mathrm{Wu}$, et al. (1989). These are given 
below and reflect the following. First, to predict secondary slip before the tensile axis overshoots the symmetry boundary, the off-diagonal components of $\left\{h_{\alpha \beta}\right\}$ (the terms that truly characterize latent hardening in single slip) must be less than the diagonal terms (active hardening), at least during stage I deformation. Secondly, the active-hardening moduli should vary at least as much as the overall hardening rate, which for the transition from stage I to stage II deformation precludes a monotonic decrease in $h_{\alpha \alpha}$ (no sum) with increasing primary slip. As a new slip system(s) becomes active the hardening rate on each system will tend to increase, the magnitude depending on the kind of dislocation locks each system forms with the newly activated one. However, the new system(s) will tend to harden much faster than the others since the existing forest obstacles outnumber the newly created ones. An analytic form for $h_{\alpha \beta}$ is proposed which satisfy these conditions.

Plastic flow of crystalline solids at low temperature has been analyzed in a time-independent continuum framework, for example, by Taylor (1938), Hill (1966), Hutchinson (1970), and Asaro and Rice (1977). The activation of slips is determined by a generalized Schmid's law (flow rule) such that any slip systems is active if and only if the corresponding shear stress and stress-rate on that system attain critical values equal to the hardness of that system. The rate of hardening of each system is related to the slip (shear) rates on all systems through the instantaneous hardening moduli $\left\{h_{\alpha \beta}\right\}$ as given by (1). For brevity, the complete single crystal constitutive equations are not given in this paper; the reader is referred to Bassani and Wu (1989) and Bassani (1990).

If the latent hardening moduli are assumed to be greater than the active ones, as proposed by Hutchinson (1970) and Asaro (1983), then the timeindependent formulation may not predict a unique set of slips (see, e.g., Hill, 1966; Hill and Rice, 1972). Although uniqueness is not an issue in a timedependent framework such as the one proposed by Asaro (1983), this alone does not address the somewhat complex nature of hardening. The new hardening description proposed guarantees uniqueness.

\section{INSTANTANEOUS HARDENING MODULI}

The least well-characterized aspect of the constitutive framework for either time dependent and independent behavior is the set of instantaneous hardening moduli $\left\{\mathrm{h}_{\alpha \beta}\right\}$ that relate the rate of hardening on each slip system to the plastic slip-rate on all systems. For FCC crystals there are 12 slip systems, many of which are activated in any deformation history. The analytical forms for $\left\{h_{\alpha \beta}\right\}$ developed below are chosen so that phenomena such as the transition from stage I to stage II hardening, the orientation dependence of hardening, latent hardening, and tensile overshoot can be reasonably predicted. To accomplish this it is necessary to incorporate a strong history dependence of slip on the instantaneous hardening moduli.

There have been many proposals for the analytical representation of $\left\{\mathrm{h}_{\alpha \beta}\right\}$. For example, the isotropic hardening law proposed by Taylor $(1938)\left(h_{\alpha \beta}=h\right)$, the independent hardening law of Koiter (1963) ( $\mathrm{h}_{\alpha \beta}=\mathrm{h} \delta_{\alpha \beta}$ where $\delta_{\alpha \beta}$ is the Kronecker delta), the geometrical hardening law proposed, the simple hardening law of Hutchinson (1970) and Asaro $(1983)\left(\mathrm{h}_{\alpha \beta}=\mathrm{qh}+(1-\mathrm{q}) \mathrm{h} \delta_{\alpha \beta}\right)$ and the hardening law of Havner and Shalaby (1977) which includes the finite rotational effect to account for the overshoot. In each case, for simplicity, $h$ has been chosen to be either a constant or a simple function that monotonically decreases with shear strain. These simplified descriptions are not consistent with many physical observations.

In Wu, et al. (1989), a careful interpretation of the transition from stage I and stage II deformation and of the orientation dependence of the $\tau-\gamma$ curve in uniaxial tension and a reinterpretation of the latent hardening experiment for FCC crystals lead to the following inequality restrictions on the instantaneous hardening moduli. With 1 denoting the primary slip system and 2 denoting the secondary system as defined, for example, in uniaxial stressing with the tensile axis within a spherical triangle: 


$$
\begin{aligned}
& \mathrm{h}_{21}\left(\gamma_{1}, 0\right)<\mathrm{h}_{11}\left(\gamma_{1}, 0\right) \\
& \mathrm{h}_{11}\left(\gamma_{1}, 0\right)<\mathrm{h}_{11}\left(\gamma_{1}, \gamma_{2}\right) \\
& \mathrm{h}_{11}\left(\gamma_{1}, \gamma_{2}\right)<\mathrm{h}_{22}\left(\gamma_{1}, \gamma_{2}<<\gamma_{1}\right)
\end{aligned}
$$

Clearly, the simple hardening rules noted above that others have adopted cannot satisfy all of these inequalities.

To describe the complex multiple slip phenomena during plastic flow, consider that the instantaneous hardening moduli depend on all the slips, i.e. $\mathrm{h}_{\alpha \beta}\left(\left\{\gamma_{\eta} ; \eta=1, N\right\}\right), \alpha, \beta=1, N$. Bassani and $\mathrm{Wu}(1989)$ proposed that

$$
\begin{aligned}
& \mathrm{h}_{\alpha \alpha}=\mathrm{F}\left(\gamma_{\alpha}\right) \mathrm{G}\left(\left\{\gamma_{\beta} ; \beta=1, \mathrm{~N}, \alpha \neq \beta\right\}\right) \quad(\text { no sum on } \alpha) \\
& \mathrm{h}_{\beta \alpha}=\mathrm{qh}_{\alpha \alpha}, \alpha \neq \beta
\end{aligned}
$$

where $F\left(\gamma_{\alpha}\right)$ is the instantaneous hardening modulus under single slip and $G$ is associated with interactive (cross) hardening where $G\left(\left\{\gamma_{\beta}=0 ; \beta=1, N\right\}\right) \equiv 1$. In general, $F$ and $G$ may be functionals of their arguments. The hardening of system $\beta$ due to slip on system $\alpha$ is simply taken to be a fraction of the active modulus $h_{\alpha \alpha}$ (no sum) through the parameter $q$. Other choices for the offdiagonal terms, e.q., the symmetric one $h_{\alpha \beta}=q\left(h_{\alpha \alpha}+h_{\beta \beta}\right)$, could easily be adopted. The one given by Eq. (4) seems natural, although not symmetric. Nevertheless, the excellent agreement with experimental observations is obtained with $q=0$, which leaves $h_{\alpha \beta}$ symmetric.

The assumption that the active hardening moduli for each slip system can be expressed as a product of the two functions (or functionals) as given in Eq. (3) can be interpreted as follows. Consider uniaxial stressing of a crystal with an initial single-slip orientation. Let $\alpha$ denote the primary slip system. Then, during stage I deformation when $\gamma_{\alpha}>0$ and $\gamma_{\beta}=0, \beta \neq \alpha$, the function $F$ characterizes the single-slip hardening behavior, namely easy glide, and $G=1$. During stage II deformation when secondary slips are activated, i.e. $\gamma_{\beta}>0$ for some $\beta \neq \alpha$, the function $G$ then characterizes the forest hardening effect caused by slip interactions between the primary system $\alpha$ and the secondary systems.

An simple form for $F$ (single slip) is

$$
F\left(\gamma_{\alpha}\right)=\left(h_{0}-h_{s}\right) \operatorname{sech}^{2}\left[\frac{\left(h_{0}-h_{s}\right) \gamma_{\alpha}}{\tau_{I} I^{-\tau_{0}}}\right]+h_{s}
$$

where $\tau_{0}$ is the initial critical resolved shear stress, $\tau_{I}$ is the stage I stress (or the breakthrough stress where large plastic flow initiates), $h_{0}$ is the hardening modulus just after initial yield, and $h_{s}$ is the hardening modulus during easy glide.

A form for $G$ (interactive hardening) that equals unity when its arguments are all zero and asymptotes to finite values when all slips $\gamma_{\beta}, \beta \neq \alpha$, are large is

$$
G\left(\left\{\gamma_{\beta} ; \beta=1, N, \beta \neq \alpha\right\}\right)=1+\sum_{\substack{\beta=1 \\ \beta \neq \alpha}}^{N} f_{\alpha \beta} \tanh \left(\frac{\gamma_{\beta}}{\gamma_{0}}\right)
$$

where $\gamma_{0}$ represents the amount of slip after which a given interaction between slip system $\alpha$ and $\beta$ reaches peak strength and each $f_{\alpha \beta}$ represent the magnitude of the strength of the interaction. For example, coplanar interactions tend to be weaker than non-coplanar ones. Values of the constants in (5) and (6) vary 
with respect to material, temperature, and prior straining history (including processing). In general, for a given material and well-defined conditions, these constants can be either directly measured from experiments or can be estimated from simulations. For example, $\tau_{0}, \tau_{I}, h_{o}$, and $h_{s}$ can be measured from uniaxial loading tests on crystals with single-slip orientations, $q$ and $\gamma_{0}$ can be estimated from the latent hardening experiments.

The amplitude factors $f_{\alpha \beta}$ in Eq. (6) depend on the type of dislocation junction (interaction) formed between slip system $\alpha$ and $\beta$, which in turn, depends on the geometric relation between the two slip systems (Franciosi, et al., 1980). For FCC crystals, these interactions are classified into five groups and $f_{\alpha \beta}$ is uniquely defined by five constants, $a_{i}, i=1,5$ :

$a_{1}$ (no junction): the resultant Burgers vector from slip system $\alpha$ and $\beta$ is parallel to the original one.

$a_{2}$ (Hirth lock): the resultant Burgers vector is not energetically admissible.

$a_{3}$ (co-planar junction): the resultant Burgers vector is on the same slip plane as the original ones.

$\mathrm{a}_{4}$ (glissile junction): the resultant Burgers vector is energetically admissible and on one of the two slip planes.

$a_{5}$ (sessile junction): the resultant Burgers vector is energetically admissible but not on either of the two slip planes.

In general, $a_{5}>a_{4}>a_{3}>a_{2}>a_{1}$ is expected. For each pair of slip systems in the FCC single crystal, the table that specifies the type of junction and relates each value of $f_{\alpha \beta}$ to one of the $a_{i}$ 's is given in Bassani and Wu (1989).

\section{CONCLUSIONS}

Predicted uniaxial stress-strain curves for single crystal copper based on the hardening law in Eqs. (3)-(6) for both "single-slip" and multiple-slip orientations of the tensile axis are plotted in Fig. 1 (see Bassani and Wu, 1989 for details). So long as the tensile axis is well within the spherical triangle the Schmid factor on the primary system is nearly 0.5 so that the uniaxial stress is approximately twice shear stress on the primary system. As the tensile axis rotates towards the [100]-[111] symmetry boundary the Schmid factor decreases. During easy-glide in stage $I$, only one slip system is active and this one hardens faster than the others. For the present case where $q=0$ in Eq. (4) the latent systems do not harden during stage I. Eventually, as the tensile stress increases the critical shear stress is exceeded on one or more secondary systems which leads to the high multi-slip hardening of stage II. Thereafter, the deformation is no longer single slip, and the tensile axis eventually overshoots the symmetry boundary as seen in experiments even though latent hardening is absent in this calculation.

The predicted curves in Fig. 1 for various orientations display the dependence of the overall hardening-rate on the initial orientation and are in reasonable agreement with experiments (see, Wu, et al., 1989 and Diehl, 1956). Note that as the orientation approaches the [100]-[111] symmetry boundary, the length of easy glide decreases whereas hardening rate increases. This is due to secondary slip(s) and the resulting slip interactions. The predicted uniaxial stress-strain curve for the high symmetry [111] orientation of the tensile axis where multi-slip commences immediately after plastic deformation shows that easy-glide and lattice rotation are both suppressed as observed in experiments. In this case, the initial high hardening rate is due to slip interactions. Furthermore, the [111] orientation has a higher hardening rate than [100] even though [111] has a fewer number of active slip systems, namely 6 versus 8 . 
This is due to different types of dislocation locks that are formed by the active slip systems. A trend towards stage III deformation (parabolic hardening) is also predicted for some orientations.

Finally, some preliminary wire texture simulations (based upon Hill's, 1965 self-consistent calculation) indicate that the nature of slip system interactions and, particularly, the dependence of the instantaneous hardening moduli on the history of slips can have a significant influence on texture evolution. For example, when latent hardening is taken to be stronger than active hardening then a given "primary" set of active slips in a particular crystal tend to persist longer than it would according to the new description with the overall level of polycrystal hardening the same. It seems that any increase in tendency for "secondary" slips, which the new description emphasizes, tends to counter the rotations associated with the "primary" set of slips, while the trends toward final orientations are similar. That is, at a given level of macroscopic strain, the new description would predict less texturing.

\section{ACKNOWLEDGEMENTS}

This research was supported by the United States Department of Energy under Grant DE-FG02-85ER45188.

\section{REFERENCES}

1. R. J. Asaro, Adv. Appl. Mech. 23, 1 (1983).

2. R. J. Asaro and J. R. Rice, J. Mech. Phys. Solids 25, 309 (1977).

3. S. J. Basinski and Z. S. Basinski, 2nd International Conference on the Strength of Metals and Alloys (ASM Metal Park, Ohio I), 189 (1970).

4. J. L. Bassani and T.-Y. Wu, "Latent hardening in single crystals, part 2, analytical characterization and predictions," University of Pennsylvania (1989).

5. J. L. Bassani, Appl. Mech. Rev. 43, S320 (1990).

6. J. Diehl, Z. Metall. 47, 331 (1956).

7. P. Franciosi, M. Berveiller and A. Zaoui, Acta Metall. 28, 273 (1980).

8. K. S. Havner and A. H. Shalaby, Proc. Roy. Soc. A358, 47 (1977).

9. R. Hill (1965), J. Mech. Phys. Solids 13, 89 (1965).

10. R. Hill, J. Mech. Phys. Solids 14, 95 (1966).

11. R. Hill and J. R. Rice, J. Mech. Phys. Solids 20, 401 (1972).

12. R. W. K. Honeycombe, The Plastic Deformation of Metals, (2nd Ed., Edward Arnold, Australia) Chapter 4.

13. J. W. Hutchinson, Proc. Roy. Soc., Series A 319, 247 (1970).

14. N. R. Joshi and R. E. Green, Jr., J. Mat. Sci. 15, 729 (1980).

15. W. Koiter, Q. Appl. Maths. 11, 350 (1953).

16. G. I. Taylor, J. Inst. Met. 62, 307 (1938).

17. T.-Y. Wu, J. L. Bassani, and C. Laird, "Latent hardening in single crystals, part 1, theory and experiments", University of Pennsylvania (1989). 